\title{
Immunomodulatory Effects of Fucoidan on NK Cells in Ovariectomized Rats
}

\author{
Seung Namkoong ${ }^{1}$, Ye-Jin Kim², Taeseong Kim² and Eun-Hwa Sohn ${ }^{2}$. \\ ${ }^{1}$ Department of Physical Therapy, Kangwon National University, Samcheok 245-710, Korea \\ ${ }^{2}$ Department of Herbal Medicine Resource, Kangwon National University, Samcheok 245-710, Korea
}

\begin{abstract}
In this study we investigated the effects of supplementation with fucoidan from brown alga on the function of natural-killer (NK) cells to evaluate the possibility as an immunomodulator in ovariectomized (OVX) rats. A total of 18 female Wistar rats (six weeks) were used this study and 12 rats were OVX, and the rest of rats were sham-operated. The sham and one OVX group were fed standard diet, and the remaining OVX group received fucoidan $(0.05 \%$ supplemented diet). After 12 weeks of supplementation, rats were sacrificed to assess the tumoricidal activity of the NK cells and the NO-iNOS regulation from splenocytes. The mass of body and the immune organs such as spleen and thymus were also studied. In OVX rats, body and thymus weights increased, however fucoidan supplementation did not change the body mass and organs weight compared to OVX group. Fucoidan supplementation increased NK cell activity and reduced NO-iNOS production in OVX rats. Ex vivo treatment of fucoidan increased NK cell activity in splenocytes from shame and OVX rats. Ex vivo, we confirmed that fucoidan partially reduced the NK cell activity in the presence of iNOS inhibitors in OVX-splenocytes. These results indicate fucoidan supplementation has a NK cell tumoricidal activity, which are regulated by the iNOS production in OVX rats. This suggests that fucoidan is useful for potential therapeutic strategies as a nutrient in regulating the NK cells in postmenopausal osteoporosis patients.
\end{abstract}

Key words - Antitumor effect, In vivo, iNOS, Osteoporosis

\section{Introduction}

Aging plays an important role in many biological functions within a body, and it is the main predictor for osteoporosis. With advancing aging and particularly with post-menopausal conditions, the activity of osteoclasts, cells that break down bone through bone resorption, is greater than that of osteoblasts, cells responsible for bone formation, in women. This ultimately gives rise to osteoporosis (Yamaguchi, 2006). Aging is also associated with a decline in immune function, known as immunosenescence, that contributes to the increased susceptibility to infection, cancer, and autoimmune diseases observed in humans (Mocchegiani and Malavolta, 2004; Sohal and Allen, 1990). Aging-related alterations of the cellular components of innate immunity might therefore be involved in the impairment of adaptive immunity observed in the elderly (Mocchegiani and Malavolta, 2004; Jang et al., 2010).

${ }^{\star}$ Corresponding author. E-mail : ehson@kangwon.ac.kr
Ovariectomy induced bone loss in rats and postmenopausal bone loss in humans share many similar characteristics, and similar skeletal response to therapy with $17 \beta$-estradiol (Kalu, 1991; Kalu et al., 1991). These similarities are strong evidence that the OVX rat bone loss model is suitable for studying the prevention and treatment of postmenopausal bone loss.

NK cells are an important component of the innate immune system and mediate cytolytic activities against tumors and virus-infected targets. Recently increasing evidences indicate that NK cells may play a pivotal role in some female predominant diseases or in normal physiological conditions. Some studies suggest that estrogen may be involved in the regulation of immunocompetence in immune system and it modulates activities of NK cells (Nakaya et al., 2006). Baral et al. also found that estrogen reduced NK cell cytotoxicity against tumor targets but this effect was dependent on duration of exposure to estrogen (Baral et al., 1995).

The brown seaweeds, which have been consumed as a food or relish to provide a dietary fiber source, are the most important 
economic seaweeds cultured in Asia. Fucoidan, which is found in extracts from brown seaweeds (e.g., Ascophyllumnodosum and Fucus vesiculosus), is a polyanionic macromolecule composed predominantly of sulfated fucose moieties. In recent years, fucoidan has been extensively studied due to its numerous biological activities including its anticoagulant, antithrombotic, antitumor, antiviral, anti-complement and anti-inflammatory effects (Patel et al., 2002; O'Leary et al., 2004; Mahony et al.,1991; Baba et al., 1988).

Although these favorable effects of fucoidan have been reported and evaluated, the effects of fucoidan on activity of NK cells in osteoporosis have not been evaluated. The estrogendeficient ovariectomized (OVX) osteoporosis model is useful for evaluation of osteoporotic drugs because several bonerelated parameters are clearly decreased by ovariectomy within 10 to 14 weeks after the operation. In this model, we examined the effects of fucoidan on the NK cell function in OVX rats and identify the possible involvement of iNOS that may mediate the regulatory effect of NK cell.

\section{Materials and Methods}

\section{Experimental animals and diets}

Female Wistar rats (six weeks) were used for the experiment. The animals were kept for 5 days before the onset of the experiment to acclimatize to our laboratory conditions (the room temperature was $25^{\circ} \mathrm{C}$ with a $12 \mathrm{~h} / 12 \mathrm{~h}$ light/dark cycle), then 12 rats were OVX and 6 rats were sham-operated under anesthesia with intraperitoneal (IP) injection of sodium pentobarbital at a dose of $30 \mathrm{mg} / \mathrm{kg}$ body weight. After operation, rats were kept in separate cages and fed standard diet before being sacrificed. To prevent hyperphagia associated with ovariectomy, the OVX rats were pair-fed to the mean intake of those in the sham group (Wronski et al., 1987). All rats were allowed free access to drinking ion-exchanged distilled water for the duration of the whole experiment. The sham and one OVX group were fed standard diet, and the remaining OVX group received fucoidan $(0.05 \%$ fucoidan modified AIN$93 \mathrm{M}$ diet) supplemented diet. We confirmed that the ovariectomy significantly decreased $(t$-test, $p<0.05)$ bone mineral density at 10 weeks after surgery. The animals were sacrificed after ether anesthesia at 12 weeks. Spleen samples were collected aseptically for the NK cell assays. The rats were observed daily for clinical signs. None of the rats showed any abnormal growth and behavior and no sign of illness. Animal care and all experimental protocols were performed following the Institute for Laboratory Animal Research (ILAR) guideline.

\section{Preparation of fucoidan and Reagents}

Unless stated otherwise, all chemicals were purchased from the Sigma Chemical Co. (St Louis, MO). Fucoidan extracted from Fucus vesiculosus was also purchased from Sigma-Aldrich (St. Louis, MO) and dissolved in distilled water. The RPMI 1640 medium and fetal bovine serum (FBS) were purchased from GIBCO (Grand Island, NY). The XTT \{2,3-Bis(2-methoxy-4-nitro-5-sulfophenyl-2H-tetrazolium5-carboxanilide inner salt cell viability assay kit was purchased from WelGENE (Daegu, South Korea). All the tissue culture reagents and fucoidan were assayed for any endotoxin contamination using the Limulus lysate test (E-Toxate, Sigma) for in vitro assay, and the endotoxin levels were found to be $<$ $10 \mathrm{pg} / \mathrm{ml}$.

\section{NK cell-mediated tumoricidal activity}

A modification of the method reported by Mosmann et al. was used (Mosmann, 1983; Namkoong et al., 2011). The spleens were aseptically removed and dissociated into a singlecell suspension in a culture medium. The concentration was adjusted to $2 \times 10^{5}$ cells $/ \mathrm{ml}$. The culture medium was RPMI 1640 containing $10 \%$ heat-inactivated FBS, penicillin (100 $\mathrm{IU} / \mathrm{ml})$ and streptomycin $(100 \mu \mathrm{g} / \mathrm{ml})$. Spleen cells from the shame and OVX rats were tested as effector cells and YAC-1 mouse lymphoma cells (ATCC, Rockville, MD) were used as the target cells. The NK cell assays were carried out in 96well v-bottom plates at effector/target (E:T) cells ratios 50:1 with $1 \times 10^{4}$ of the target cells in a final well volume of $200 \mu \mathrm{l}$ at $37^{\circ} \mathrm{C}$ for $4 \mathrm{~h}$ in a $5 \% \mathrm{CO}_{2}$ humidified incubator. After incubation of cells for $4 \mathrm{~h}, 20 \mu \mathrm{l}$ of phenazine methosulphate (PMS); electron-coupling reagent and $25 \mu \mathrm{l}$ of XTT were added to each well. The cells were further incubated for $3 \mathrm{~h}$ to allow XTT formazan production. The absorbance were determined with a microplate reader at a test wavelength of $450 \mathrm{~nm}$ and a reference wavelength of $690 \mathrm{~nm}$. 


\section{Westem blot analysis}

The amount of iNOS was measured by Western blot analysis. Rats were immediately sacrificed and isolate spleen. After homogenized of spleen tissue and lysed in RIPA buffer. Protein concentrations were measured using the DC Protein Assay (Bio-Rad Laboratories, Hercules, CA). Twenty micrograms of each sample was electrophoresed on $10 \%$ sodium dodecylsulfate polyacrylamide gels and transferred to Hybond-ECL nitrocellulose membranes (Amersham Biosciences, Piscataway, NJ). The membranes were blocked with $5 \%$ skim milk in TBS-T for $1 \mathrm{~h}$. The membranes were incubated with primary antibody against iNOS for $24 \mathrm{~h}$. They were then washed with TBS-T once for $15 \mathrm{~min}$ and three times for $5 \mathrm{~min}$ and incubated with secondary HRP-conjugated anti-rabbit antibody for $1 \mathrm{~h}$. The membranes were washed again as described above. Autoradiography was carried out using an enhanced chemiluminescence kit.

\section{Statistical analysis}

Each experiment was repeated at least two times, and the results of one representative experiment are shown. The results were expressed as means \pm S.E.M. and analyzed via Student's $\mathrm{t}$ test and ANOVA. The significant values are represented by an asterisk $\left({ }^{*}, * 00.05\right.$ and $\left.{ }^{* *}{ }^{* \#} p<0.01\right)$.

\section{Results and Discussion}

The most characteristic function of NK cells is non-MHC restricted killing of target cells, such as tumor, and thus NK cells are important for maintaining health in body (Borrego et al., 1999). It has been reported that aging attributed to various factors of NK cells. For example, NK cells exhibited decreased responsiveness to its positive modulator, IL-2, in aged group (Miller, 1991) and this explains the high frequency of cancer and infectious diseases with aging. Osteoporosis is one of the aging-related diseases (Yamaguchi, 2006). With advancing aging and particularly after menopause in women, the activity of osteoclasts, cells that break down bone, is greater than that of osteoblasts, cells that build bone. In this study, we determined the tumoricidal activity of the NK cells in OVX and the regulatory effects of the supplementation with edible seaweed fucoidan. We showed that the increasing effects on NO-iNOS production and the suppressive effect of NK cells tumoricidal activity in OVX rats compared to sham group. Furthermore, fucoidan supplementation in OVX group recovered all these effects such as NO-iNOS production and NK cells tumoricidal activity (Fig. 1-3).

Recently it has been reported that NK cell tumoricidal activity could be regulated by sex hormones. Yovel et al. found that there was an inhibition of specific lysis of Yac-1

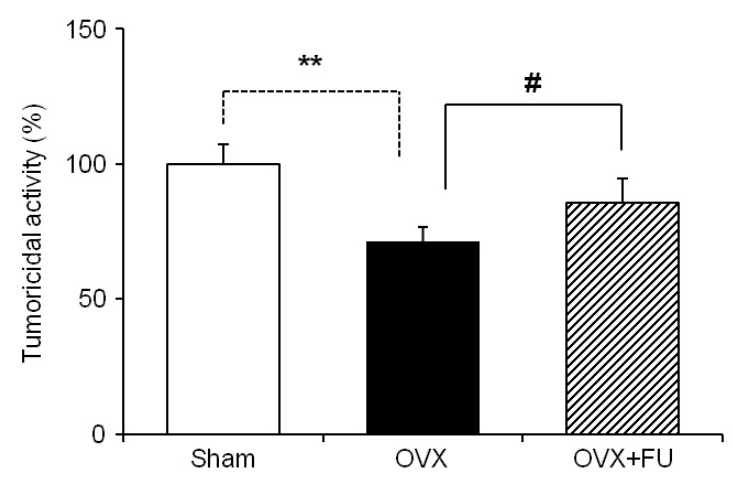

Fig. 1. Effect of the fucoidan (FU) supplementation on the NK cells tumoricidal activity from experimental rats. Splenocytes were cultured with Yac-1 tumor cells for $4 \mathrm{~h}$. The NK cells tumoricidal activity examined at effecter/target cells ratio of $50: 1$ by XTT assay. The data is expressed as a means \pm S.E.M of quadruplicates of a representative experiment. ${ }^{* *} P<0.01$ : significantly different from the sham group. ${ }^{\#} P<0.05$ : significantly different from the OVX group.

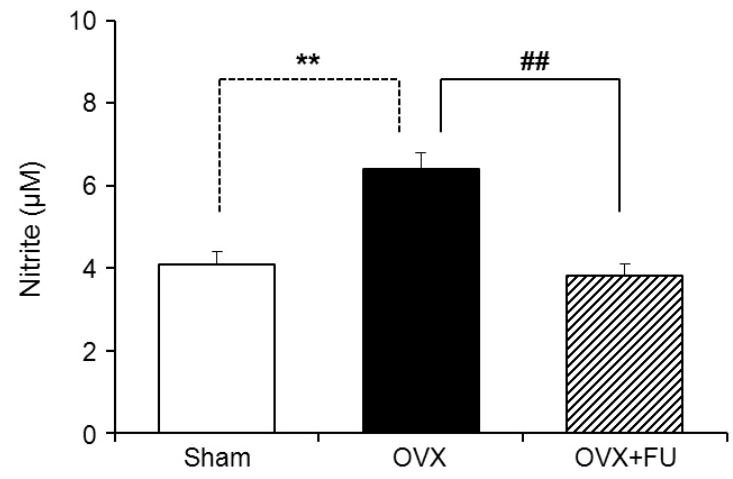

Fig. 2. The effects of the fucoidan (FU) supplementation on the production of NO in splenocytes from experimental rats. These results are means \pm S.E.M of quintuplicates from a representative experiment. ${ }^{* *} P<0.01$ : significantly different from the sham group. ${ }^{\#} P<0.01$ : significantly different from the OVX group. 


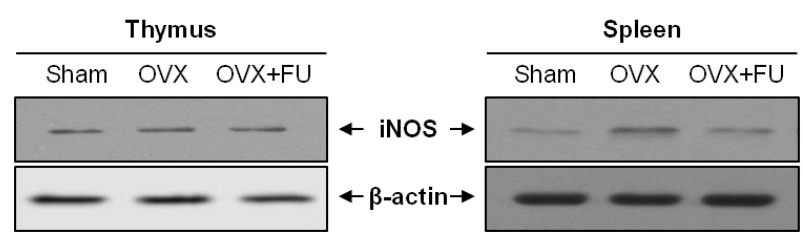

Fig. 3. The effects of the fucoidan (FU) supplementation on the expression iNOS in splenocytes and thymocytes from experimental rats. Spleen and thymus tissue lysate were determined by Western Blot assay.
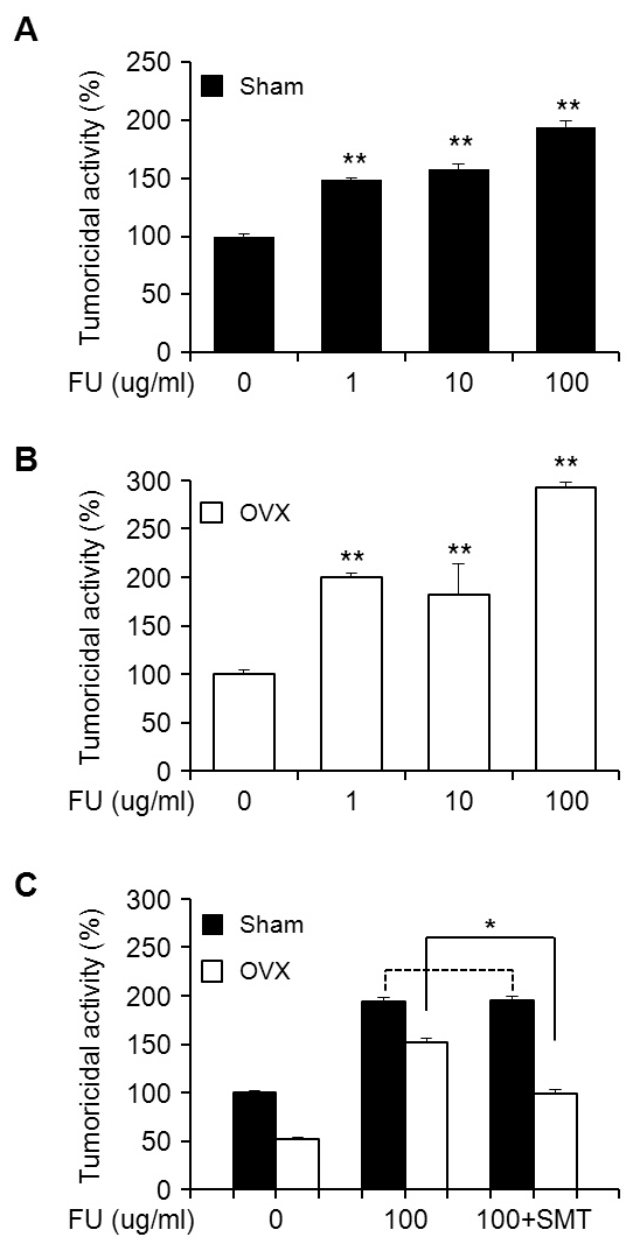

Fig. 4. The effects of fucoidan (FU) on the NK cell tumoricidal activity in the splenocytes from sham (A), OVX group (B) and the NK cell tumoricidal activity of $\mathrm{FU}$ in the absence or presences of s-methylisothiourea (SMT; $100 \mu \mathrm{M}$ ), iNOS inhibitors (C). Splenocytes $\left(5 \times 10^{4}\right.$ cells/well $)$ were treated with various FU $(1-100 \mu \mathrm{g} / \mathrm{ml}$ or $100 \mu \mathrm{g} / \mathrm{ml})$ or in the presence of SMT $4 \mathrm{~h}$. The turmoridical activity was examined at E:T ratio of 50:1. The data represents the means \pm S.E.M. of quadruplicate experiments. ${ }^{*} p<0.01,{ }^{*} p<0.05$ : significantly different from the control or FU alone treated cells. target after a long-term exposure of the NK cells to $17 \beta$ -estradiol (Yovel et al., 2001). In addition, several data in animal model studies also provided direct evidence that chronic administration of high doses of $17 \beta$-estradiol to mice resulted in a decreased activity of NK cells (Yovel et al., 2001). Thus, estrogen imbalance level caused change of NK cell cytotoxicity and the low NK activity induced by IL-2 was found to be a predictor of morbidity for various infection and cancer in osteoporosis (Levy et al., 1991; Kim et al., 2009).

To examine whether the effect of fucoidan on NK cell tumoricidal activity is mediated by NO production which is involved in iNOS expression, the splenocytes from sham and OVX rats were treated with fucoidan $(100 \mu \mathrm{g} / \mathrm{ml})$ in the absence or presence of s-methylisothiourea, preferential inhibitor of iNOS. As shown in Fig. 4, the treatment of fucoidan resulted in a significant enhancement of NK cell activity in splenocyte from sham and OVX rats in dose dependant manners. Moreover it appears that iNOS expression was involved in the regulation of NK cell activity in fucoidan-treated OVX splenocytes, whereas inhibition of iNOS has no effects of the NK cells activity in fucoidan-treated sham cells. These data imply that the estrogen-deficient stress activates innate immune cells such as NK cells and changed intracellular NO levels. Thus it is speculated that fucoidan and cytokines produced by these cells could exert combinatory effects on NK cell activities in OVX state.

In previous study, red ginseng acidic polysaccharide (RGAP) alone had no effect on the function of macrophages for killing tumor cells. However, a combination of RGAP and recombinant IFN- $\gamma$ (rIFN- $\gamma$ ) enhanced their killing of tumor cells (Choi et al., 2008). There was a report that IFN- $\gamma$ was a major product of murine NK cells during pregnancy and its production was enhanced by a long-term exposure of cells to estradiol. Therefore, we could postulate that hormonal imbalance such as estrogen-deficiency changed the secretion of various cytokines of NK cells. Moreover, the fucoidan exerted combinatory influence on such cytokines regulation for NK cell activities, and it was regulated by secondary signals such as NO-iNOS production.

In our experimental systems using OVX rat model, as shown in Table 1, ovariectomy significantly increased the body mass and thymus weight index but not spleen compared 
Table 1. Effects of fucoidan supplement on the body mass and index of immune organs in ovariectomized (OVX) rats

\begin{tabular}{lccc}
\hline \hline Organ / Body weight & \multicolumn{1}{c}{ Sham } & OVX & OVX+FU \\
\hline Thymus $^{\dagger}$ & $0.15 \pm 0.005$ & $0.24 \pm 0.009^{\mathrm{a}}$ & $0.24 \pm 0.011$ \\
Spleen $^{\dagger}$ & $0.28 \pm 0.012$ & $0.27 \pm 0.008$ & $0.28 \pm 0.007$ \\
Body weight $(\mathrm{g})$ & $268.5 \pm 3.7$ & $330.25 \pm 6.5^{\mathrm{a}}$ & $325.75 \pm 8.3$ \\
\hline
\end{tabular}

${ }^{\dagger}$ Index $=($ organ mass $(\mathrm{g}) /$ body mass $(\mathrm{g})) \times 100, \mathrm{FU}$ : fucoidan, ${ }^{\mathrm{a}} \mathrm{P}<0.01$ statistically significant differences in comparison with the sham control rats.

to the sham rats $(p<0.01)$. However, fucoidan supplementation did not affect the changes of the body weight and thymus/spleen weight index of the OVX rat.

Collectively, we could suggest that fucoidan supplementation have a possibility as a potential immunotherapeutic agent for osteoporotic patients. Though we do not know whether iNOS expression is involved in NK cell activities in details, our data demonstrate that the effect of fucoidan on NK cellmediated tumoricidal activity is regulated by iNOS expression and fucoidan provides secondary signals for the regulatory induction of NK cell activities in OVX states. The precise mechanism of estrogen in modulating NK cells and the exact role of NK cells in the osteoporosis remain unclear. In addition, the exact explanation for modulation of NK activity by fucoidan in OVX rats is not known yet. Therefore we need to examine further the effects of fucoidan on the regulation of NOiNOS and the level of cytokines such as IL-2 and IFN- $\gamma$, which are may necessary for activation and maturation of NK cells.

In summary, our data imply that the estrogen-deficient stress activates innate immune cells such as NK cells and changed the secretion of NO and iNOS expression, and these were regulated by fucoidan supplementation. This suggests that fucoidan is useful for potential therapeutic strategies as a nutrient in regulating the NK cells in postmenopausal osteoporosis patients.

\section{Literature Cited}

Baba, M., R. Snoeck, R. Pauwels and E. de Clercq. 1988. Sulfated polysaccharides are potent and selective inhibitors of various enveloped viruses, including herpes simplex virus, cytomegalovirus, vesicular stomatitis virus, and human immunodeficiency virus. Antimicrob. Agents Chemother. 32:
1742-1745.

Baral, E., E. Nagy and I. Berczi. 1995. Modulation of natural killer cell-mediated cytotoxicity by tamoxifen and estradiol. Cancer 75:591-599.

Borrego, F., M.C. Alonso, M.D. Galiani, J. Carracedo, R. Ramirez, B. Ostos, J. Pena and R. Solana. 1999. NK phenotypic markers and IL-2 response in NK cells from elderly people. Exp. Gerontol. 34:253-265.

Choi, H.S., K.H. Kim, E. Sohn, J.D. Park, B.O. Kim, E.Y. Moon, D.K. Rhee and S. Pyo. 2008. Red ginseng acidic polysaccharide (RGAP) in combination with IFN- $\gamma$ results in enhanced macrophage function through activation of the NF- $\kappa$ B pathway. Biosci. Biotechnol. Biochem. 72:1817-1825.

Jang, S.A., S. Namkoongm and E.H. Sohn. 2010 Effects of $\beta$ -glucans from Coriolus versicolor on macrophage functions in young and aged mice. J. Korean Plant Res. 23(6): 498502.

Kalu, D.N. 1991. The ovariectomized rat model of postmenopausal bone loss. Bone Miner. 15:175-192.

Kalu, D.N., C.C. Liu, E. Salemo, B. Hollin, R. Echon and M. Ray. 1991. Skeletal response of ovariectomized rats to low and high doses of 17 $\beta$-estradiol. Bone Miner. 14:175-187.

Kim, K.S., S. Pyo and E.H. Sohn. 2009. Immunomodulation of NK Cell activity by red ginseng acidic polysaccharide (RGAP) in ovariectomized rats. J. Ginseng Res. 33(2):99-103.

Levy, S.M., J. Fernstrom, R.B. Herberman, T. Whiteside, J. Lee, M. Ward and M. Massoudi. 1991. Persistently low natural killer cell activity and circulating levels of plasma beta endorphin: risk factors for infectious disease. Life Sci. 48:107-116.

Mahony, M.C., S. Oehninger, G.F. Clark, A.A. Acosta and G.D. Hodgen. 1991. Fucoidin inhibits the zona pellucidainduced acrosome reaction in human spermatozoa. Contraception. 44:657-665.

Miller, R.A. 1991. Aging and immune function. Int. Rev. Cytol. 124:187-215 
Mocchegiani, E. and M. Malavolta. 2004. NK and NKT cell functions in immunosenescence. Aging Cell. 3:177-184.

Mosmann, T. 1983. Rapid colorimetric assay for cellular growth and survival: application to proliferation and cytotoxicity assays. J. Immunol. Methods 65:55-63.

Nakaya, M., H. Tachibana and K. Yamada. 2006. Effect of estrogens on the interferon- $\gamma$ producing cell population of mouse splenocytes. Biosci. Biotechnol. Biochem. 70:47-53.

Namkoong S., E.S. Cheoung, H. Joo, S.A. Jang, Y.J. Yang, S.C. Kang, K.H. Jang and E.H. Sohn. 2011. Immunomodulatory effects of Eisenia bicyclis on innate immune cells in acute exercise-stress rat model. J. Korean Plant Res. 24(3):286-291.

O'Leary, R., M. Rerek and E.J. Wood. 2004. Fucoidan modulates the effect of transforming growth factor (TGF)- $\beta 1$ on fibroblast proliferation and wound repopulation in in vitro models of dermal wound repair. Biol. Pharm. Bull. 27:266270 .

Patel, M.K., B. Mulloy, K.L. Gallagher, L. O'Brien and A.D.
Hughes. 2002. The antimitogenic action of the sulphated polysaccharide fucoidan differs from heparin in human vascular smooth muscle cells. Thromb. Haemost. 87:149-154.

Sohal, R.S. and R.G. Allen. 1990. Oxidative stress as a causal factor in differentiation and aging: a unifying hypothesis. Exp. Gerontol. 25:499-522.

Wronski, T.J., P.A. Schenk, M. Cintron and C.C. Walsh. 1987. Effect of body weighton osteopenia in ovariectomized rats. Calcif. Tissue Int. 40:155-159.

Yamaguchi, M. 2006. Regulatory mechanism of food factors in bone metabolism and prevention of osteoporosis. Yakugaku Zasshi. 126:1117-1137.

Yovel, G., K. Shakhar and S. Ben-Eliyahu. 2001. The effects of sex, menstrual cycle, and oral contraceptives on the number and activity of natural killer cells. Gynecol. Oncol. 81:254262.

(Received 29 May 2012 ; Revised 14 June 2012 ; Accepted 25 June 2012) 